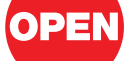

SUBJECT AREAS:

QUANTUM PHYSICS

MAGNETIC MATERIALS AND

DEVICES

ATOMIC AND MOLECULAR

PHYSICS

QUANTUM OPTICS

Received

22 August 2012

Accepted

28 August 2012

Published

13 September 2012

Correspondence and requests for materials should be addressed to B.D. (bogdan. damski@gmail.com)

\section{A quantum phase transition in a quantum external field: Superposing two magnetic phases}

\author{
Marek M. Rams ${ }^{1,2,3}$, Michael Zwolak ${ }^{4}$ \& Bogdan Damski ${ }^{1}$ \\ 'Los Alamos National Laboratory, Theoretical Division, MS B2 13, Los Alamos, New Mexico, 87545, USA, ${ }^{2}$ Vienna Center for \\ Quantum Science and Technology, Faculty of Physics, University of Vienna, Vienna, Austria, ${ }^{3}$ Institute of Physics, Jagiellonian \\ University, Reymonta 4, 30-059 Kraków, Poland, ${ }^{2}$ Department of Physics, Oregon State University, Corvallis, OR 97331 , USA.
}

We study an Ising chain undergoing a quantum phase transition in a quantum magnetic field. Such a field can be emulated by coupling the chain to a central spin initially in a superposition state. We show that - by adiabatically driving such a system - one can prepare a quantum superposition of any two ground states of the Ising chain. In particular, one can end up with the Ising chain in a superposition of ferromagnetic and paramagnetic phases - a scenario with no analogue in prior studies of quantum phase transitions.

Remarkably, the resulting magnetization of the chain encodes the position of the critical point and universal critical exponents, as well as the ground state fidelity.

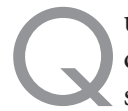
uantum phase transitions (QPTs) occur when dramatic changes in the ground state properties of a quantum system are induced by a tiny variation of an external parameter, such as the magnetic field in spin systems ${ }^{1}$ or the intensity of a laser beam in cold atom simulators of Hubbard-like models ${ }^{2}$. In all current studies of QPTs, the external parameter is assumed to be classical, i.e., it has a well-defined instantaneous value. However, the field inducing a QPT can be quantum as well, taking on different values by virtue of being in a superposition of states. In fact, tremendous progress with the preparation and manipulation of cold atom/ion systems will allow for creation of scenarios where the quantum nature of the "external" parameter will play a central role.

For instance, cavity-QED systems offer intriguing possibilities to study quantum control parameters ${ }^{3-5}$. In these systems, photons - bouncing off two parallel mirrors - interact with ultracold atoms. If the number of photons in the cavity does not fluctuate, atoms experience an "external" periodic potential $\cos ^{2}(\mathbf{k x})$, whose amplitude is proportional to the number of intra-cavity photons ( $k$ is the photon wave-vector). Atoms in such a system would be either in the superfluid phase or in the Mott insulator phase ${ }^{2}$. It may be possible, however, to create a coherent superposition of the intra-cavity photonic states, giving rise to quantum fluctuations in the number of photons between the mirrors. The atoms would then be exposed to a coherent superposition of periodic potentials with the same period but differing amplitudes. In this case, one can have atoms in a superposition of two quantum phases, i.e., simultaneously in superfluid and Mott insulator ground states ${ }^{3}$. Such a situation has no counterpart in traditional studies of QPTs where the system is either in one phase or another.

An analogous phenomenon can be envisioned in central spin models. These models are used to describe qubit environment interactions in nitrogen-vacancy centers in diamond ${ }^{6}$, quantum dots in semiconductors ${ }^{7,8}$, NMR experiments ${ }^{9}$, etc. The focus is typically on the loss of coherence of the qubit while ignoring the environmental degrees of freedom. We will take the opposite perspective and explore the quantum state of the environment subjected to an effective quantum potential originating from the central spin. For an experimental study of such a scenario, one needs a well-controlled system, which we expect will be delivered in the foreseeable future by ion simulators of spin chains ${ }^{10,11}$.

\section{Results}

The model. We will discuss the most striking consequence of a QPT in a quantum potential: The possibility of having the system in a superposition of ground states belonging to different phases, as shown in Fig. 1. We consider a quantum Ising chain uniformly coupled to a (central) spin-1/2 (Fig. 2): 


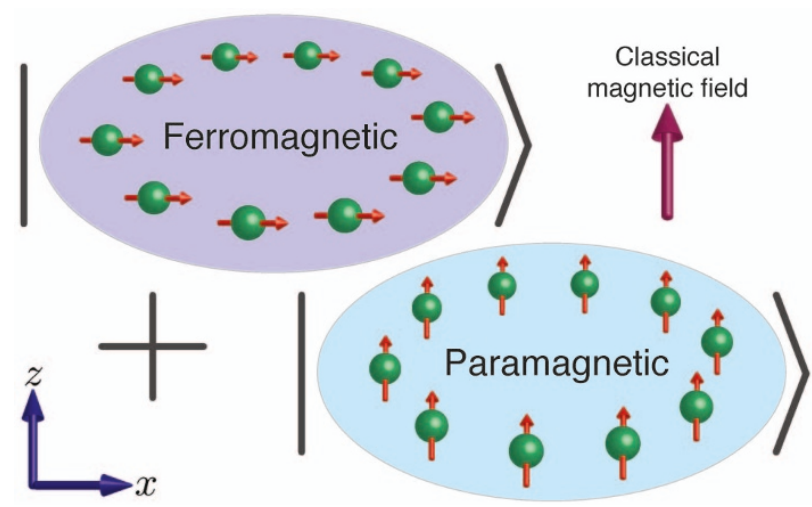

Figure 1 Schematic of a superposition of two different quantum phases in an Ising chain. In the paramagnetic phase spins try to align with the classical magnetic field, which is oriented in the $z$ direction here. In the ferromagnetic phase spin interactions try to align spins perpendicular to the field; see the Hamiltonian (1) for details. One can prepare such a state by adiabatically evolving the chain in the presence of a central spin followed by a measurement of the spin.

$$
\hat{H}=-\sum_{n=1}^{N}\left(\sigma_{n}^{x} \sigma_{n+1}^{x}+\hat{g} \sigma_{n}^{z}\right),
$$

where $N \gg 1$ is the number of spins arranged on a periodic ring. The central spin contribution is contained in the effective magnetic field operator

$$
\hat{g}=g+\delta \sigma_{S}^{z}
$$

where $g$ is the (classical) magnetic field strength and $\delta \sigma_{S}^{z}$ is the quantum component of the field generated by an Ising coupling to the central spin $(0<\delta \ll 1)$. Without the coupling to the central spin, i.e., when $\hat{H}(g, \delta=0) \equiv \hat{H}_{I}(g)$, the Ising chain in the ground state is either in the ferromagnetic phase $(|g|<1)$ or in the paramagnetic phase $(|g|>1)$, with critical points at $g_{c}= \pm 1$. We consider below $\mathrm{g}>0$.

The successful implementation of a recent proposal simulating arbitrarily-connected spin models in linear ion chains ${ }^{11}$ will put the system we consider within experimental reach. It is interesting to note that there is no need to arrange ions, i.e., effective spins, on the ring to simulate the Hamiltonian (1), see Fig. 2(a). So far the simulation of an $N=6$ Ising chain with long-range interactions between the effective spins- $1 / 2$ has been demonstrated ${ }^{12}$, analogous to that

(a)
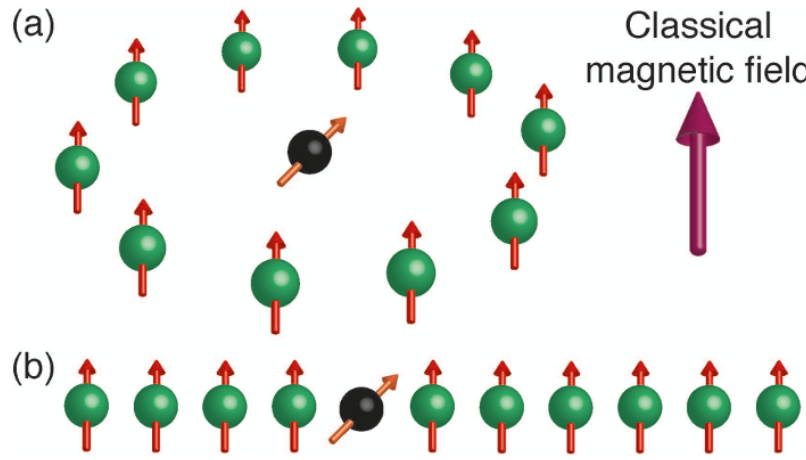

Figure $2 \mid$ Schematics of potential spin arrangements. (a) The central spin model in a classical magnetic field: The central spin is equally coupled to all the spins-1/2 arranged on a ring. (b) Possible realization of the central spin model in a linear ion chain. The ions emulate the effective spins-1/2. The couplings between the effective spins- $1 / 2$ are optically engineered to be the same as in the (a) panel. One of the ions is differently coupled to the rest of the chain to play the role of the effective central spin. The effective magnetic field is also optically engineered. shown in Fig. 2(b). The proposal is scalable and it is expected to allow for quantum simulation of models with $N \gg 1$ effective spins.

Preparation of the superposition state. QPTs can be studied either by diagonalizing the Hamiltonian for a fixed set of coupling parameters or by adiabatically evolving the system from an easyto-prepare ground state, which is especially relevant in cold atom experiments ${ }^{2}$. We take the latter approach, as the former will always force the central spin to point in either $+z$ or $-z$ direction for any $g \neq$ 0 because $\left[\hat{H}, \sigma_{S}^{z}\right]=0$.

We assume that at $t=t_{i}$ the chain is prepared in a ground state and its coupling to the central spin is turned off, which provides freedom to engineer the state of the central spin. The composite wave function is $\left|\psi\left(g\left(t_{i}\right)\right)\right\rangle=|S\rangle\left|g\left(t_{i}\right)\right\rangle$, where $|g\rangle$ is a ground state of $\hat{H}_{I}(g)$ and the central spin state is

$$
|S\rangle=c_{\uparrow} e^{i \phi_{\uparrow}\left(t_{i}\right)}|\uparrow\rangle+c_{\downarrow} e^{i \phi_{\downarrow}\left(t_{i}\right)}|\downarrow\rangle, c_{\uparrow}^{2}+c_{\downarrow}^{2}=1,
$$

where $c_{\uparrow, \downarrow}>0$. By changing both the bias field $g$ and the coupling $\delta$, the wave-function evolves according to

$$
|\psi(g(t))\rangle=\hat{T} \exp \left(-i \int_{t_{i}}^{t} d t \hat{H}[g(t), \delta(t)]\right)\left|\psi\left(t_{i}\right)\right\rangle,
$$

where $\hat{T}$ is the time-ordering operator.

As was shown in Ref. [13], $|\psi(g(t))\rangle$ can be simplified. Considering adiabatic evolution, we obtain

$$
|\psi(g(t))\rangle=e^{i \phi_{\uparrow}(t)} \mathcal{c}_{\uparrow}|\uparrow\rangle|g+\delta\rangle+e^{i \phi_{\downarrow}(t)} c_{\downarrow}|\downarrow\rangle|g-\delta\rangle .
$$

We thus study finite, i.e., gapped, systems so that adiabatic evolution is possible by changing $g(t)$ and $\delta(t)$ slow enough. In the state (3), the chain experiences an average magnetic field

$$
\langle\hat{g}\rangle=g+\delta\left(c_{\uparrow}^{2}-c_{\downarrow}^{2}\right)
$$

with fluctuations

$$
\sqrt{\left\langle\hat{g}^{2}\right\rangle-\langle\hat{g}\rangle^{2}}=2 \delta c_{\uparrow} c_{\downarrow}
$$

In particular, this shows that once the desired coupling $\delta$ is adiabatically reached, fluctuations of the quantum potential are fixed.

The state (3) is already a Schrödinger's cat state, where the two "macroscopically" distinct possibilities are the ferromagnetic and paramagnetic phases, both of which are coupled to the auxiliary two-level system. Since we are interested in the QPT of the Ising chain, we will "trace out" the central spin by measuring its state. If we will do the measurement in the $\{|\uparrow\rangle,|\downarrow\rangle\}$ basis, the superposition will be destroyed and the state of the Ising chain will be one of the ground states $|g \pm \delta\rangle$. Measurement in any other basis will result in a superposition of Ising ground states at different magnetic fields.

We assume that the measurement will be done in the eigenbasis of the $\sigma_{S}^{x}$ operator:

$$
|+\rangle=\frac{|\uparrow\rangle+|\downarrow\rangle}{\sqrt{2}}, \quad|-\rangle=\frac{|\uparrow\rangle-|\downarrow\rangle}{\sqrt{2}} .
$$

In this basis,

$$
\begin{array}{r}
|\psi(g(t))\rangle=|+\rangle \frac{\mathcal{c}_{\uparrow} e^{i \phi_{\uparrow}}|g+\delta\rangle+c_{\downarrow} e^{i \phi_{\downarrow}}|g-\delta\rangle}{\sqrt{2}}+ \\
|-\rangle \frac{c_{\uparrow} e^{i \phi_{\uparrow}}|g+\delta\rangle-c_{\downarrow} e^{i \phi_{\downarrow}}|g-\delta\rangle}{\sqrt{2}},
\end{array}
$$

where we write $\phi_{\downarrow, \uparrow}$ as shorthand for $\phi_{\downarrow, \uparrow}(t)$. Therefore, the measurement of the central spin in the state $| \pm\rangle$ leaves the chain in the state 


$$
\frac{c_{\uparrow} e^{i \phi_{\uparrow}}|g+\delta\rangle \pm c_{\downarrow} e^{i \phi_{\downarrow}}|g-\delta\rangle}{\sqrt{1 \pm 2 c_{\uparrow} c_{\downarrow} \cos \left(\phi_{\uparrow}-\phi_{\downarrow}\right) \mathcal{F}(g, \delta)}},
$$

where $\mathcal{F}(g, \delta)=\langle g-\delta \mid g+\delta\rangle$ is a ground state fidelity ${ }^{14}$, or simply fidelity, whose crucial role in this problem will be carefully discussed below. Without loss of generality, we define it in such a way that $\mathcal{F}(g, \delta)>0$.

Now we comment on the measurement of the central spin. The state $| \pm\rangle$ will occur with probability

$$
P_{ \pm}\left(\phi_{\uparrow}-\phi_{\downarrow}\right)=\frac{1}{2} \pm c_{\uparrow} c_{\downarrow} \cos \left(\phi_{\uparrow}-\phi_{\downarrow}\right) \mathcal{F}
$$

which depends on the relative phase between the ground states in the superposition (3). Since the point of the measurement is to prepare a well-defined superposition state of the Ising chain, we will describe properties of the Ising chain after finding the central spin in, e.g., the $|+\rangle$ state. Then, the Ising chain will be in the state

$$
\mid \text { Ising }\rangle=\frac{c_{\uparrow} e^{i \phi_{\uparrow}}|g+\delta\rangle+c_{\downarrow} e^{i \phi_{\downarrow}}|g-\delta\rangle}{\sqrt{1+2 c_{\uparrow} c_{\downarrow} \cos \left(\phi_{\uparrow}-\phi_{\downarrow}\right) \mathcal{F}(g, \delta)}} .
$$

This state is the desired superposition of ferromagnetic and paramagnetic ground states when

$$
g-\delta<g_{c}<g+\delta,
$$

which is depicted in Fig. 1. We propose to call such a state a Schrödinger magnet. The possibility to create such a novel state of matter is offered by the quantum magnetic field in Eq. (2). Indeed, if there would be no quantum component in the magnetic field, the wave function of the Ising chain after the adiabatic evolution would correspond to either a ferromagnetic or a paramagnetic phase ground state, but never to a superposition of both.

Ising chain in the superposition state. For simplicity, we assume that the measurements on the Ising chain are performed immediately after measuring the central spin. The expectation value of an operator $\hat{O}$ in the state (5) is

$$
O=\langle\text { Ising }|\hat{O}| \text { Ising }\rangle=\frac{O^{s}+2 c_{\uparrow} c_{\downarrow} \cos (\Delta) O^{+-}}{1+2 c_{\uparrow} c_{\downarrow} \cos (\Delta) \mathcal{F}},
$$

where $\Delta=\phi_{\uparrow}-\phi_{\downarrow}$ and

$$
O^{s}=c_{\uparrow}^{2} O^{++}+c_{\downarrow}^{2} O^{--}, O^{ \pm \pm}=\langle g \pm \delta|\hat{O}| g \pm \delta\rangle,
$$

is the "standard" average, and

$$
\mathrm{O}^{+-}=\langle g+\delta|\hat{O}| g-\delta\rangle
$$

designate the cross term that arises. For clarity of presentation, we restrict ourselves to real $\mathrm{O}^{+-}$in Eq. (6), because $\mathrm{O}^{+-}$is always real for the operators $\hat{O}=\sigma_{n}^{z}, \sigma_{n}^{x}, \sigma_{n}^{x} \sigma_{n+1}^{x}$ that we study. It is not real, however, for all operators (e.g., for $\hat{O}=\sigma_{h}^{y}$ ), and it is a straightforward exercise to extend our calculations to these cases. While the standard average does not yield any new information, the cross term provides a non-trivial correction absent in a quantum phase transition in a classical field.

To further simplify the discussion, we average Eq. (6) over several realizations where the appearance of the relative phase $\Delta$ of the superposition (3) is given by some probability distribution $p(\Delta)$. For example, such averaging may appear due to preparation of the central spin with random initial phases $\phi_{\uparrow, \downarrow}\left(t_{i}\right)$. We assume below for simplicity that $p(\Delta)=\frac{1}{2 \pi}$ for $\Delta \in[0,2 \pi)$. We denote the result of such averaging as $\bar{O}$, and define its variance through $\operatorname{var}(O)=$ $\overline{O^{2}}-\bar{O}^{2}$. Finally, we introduce the notation

$$
O_{\mathcal{F}}^{+-}=O^{+-} / \mathcal{F},
$$

because for the operators $\hat{O}$ that we consider, $O_{\mathcal{F}}^{+-}$are well-defined non-zero quantities in the thermodynamic limit in which fidelity typically tends to zero (see the Discussion section).

The phase-averaged observable and its variance are

$$
\begin{aligned}
\bar{O}=\frac{\int_{0}^{2 \pi} d \Delta p(\Delta) P_{+}(\Delta) O}{\int_{0}^{2 \pi} d \Delta p(\Delta) P_{+}(\Delta)}=O^{s}, \\
\operatorname{var}(O)=\frac{\int_{0}^{2 \pi} d \Delta p(\Delta) P_{+}(\Delta) O^{2}}{\int_{0}^{2 \pi} d \Delta p(\Delta) P_{+}(\Delta)}-\left(O^{s}\right)^{2} \\
=\left(O^{s}-O_{\mathcal{F}}^{+-}\right)^{2}\left(\frac{1}{\sqrt{1-x^{2}}}-1\right),
\end{aligned}
$$

with $x=2 c_{\uparrow} \mathcal{c}_{\downarrow} \mathcal{F}$. By expanding

$$
\frac{1}{\sqrt{1-x^{2}}}-1 \approx \frac{x^{2}}{2}
$$

we see that the square root of variance is proportional to fidelity when $x \ll 1$. The role of fidelity in our problem is discussed in the Discussion section. In the following, we use the exact solution of the Ising model to study expectation values of different observables in the superposition state (5), see the Methods section for details.

We start by looking at $\hat{O}=\hat{M}_{z}=\sigma_{n}^{z} \cdot M_{z}^{ \pm \pm}$terms have been calculated in Ref. [15]

$$
M_{z}^{ \pm \pm}=\frac{1+g \pm \delta}{\pi(g \pm \delta)} E\left(\chi_{ \pm}\right)+\frac{-1+g \pm \delta}{\pi(g \pm \delta)} K\left(\chi_{ \pm}\right),
$$

where $\chi_{ \pm}=4(g \pm \delta) /(1+g \pm \delta)^{2}$, and $K$ and $E$ are elliptic functions of the first and the second kind, respectively. Above a large $N$ limit is assumed to simplify the expressions (see the Methods section for the exact finite $N$ expressions).

The cross terms can be obtained from the eigenequation

$$
\hat{H}_{I}(g \pm \delta)|g \pm \delta\rangle=N \varepsilon(g \pm \delta)|g \pm \delta\rangle,
$$

where $\varepsilon$ is the ground state energy per $\operatorname{spin}^{15}$. Indeed, one gets from it

$$
M_{z}^{+-}=\mathcal{F}(g, \delta) \frac{\varepsilon(g-\delta)-\varepsilon(g+\delta)}{2 \delta} .
$$

In the limit of $N \rightarrow \infty, \varepsilon(g \pm \delta)=-\frac{2}{\pi}(1+g \pm \delta) E\left(\chi_{ \pm}\right)$. Consequently,

$$
M_{z \mathcal{F}}^{+-}=\frac{1+g+\delta}{\pi \delta} E\left(\chi_{+}\right)-\frac{1+g-\delta}{\pi \delta} E\left(\chi_{-}\right)
$$

The dependence of magnetization on the relative phase of the superposition and the variance of magnetization at $g=1$ are depicted in Fig. 3.

We mention in passing that similar expressions can be obtained for $\hat{O}=\hat{C}_{x}=\sigma_{n}^{x} \sigma_{n+1}^{x}$. Indeed, it is known from Ref. [15] that

$$
C_{x}^{ \pm \pm}=\frac{1+g \pm \delta}{\pi} E\left(\chi_{ \pm}\right)+\frac{1-g \mp \delta}{\pi} K\left(\chi_{ \pm}\right)
$$

and one can use again Eq. (7) to derive

$$
C_{x}^{+-}=\frac{\mathcal{F}(g, \delta)}{2}\left[\frac{g-\delta}{\delta} \varepsilon(g+\delta)-\frac{g+\delta}{\delta} \varepsilon(g-\delta)\right] .
$$

Since these results are analogous in structure to the ones already discussed, we will not analyze them.

Next, we study spontaneous magnetization in the $x$-direction. The system will acquire such a magnetization when a tiny field breaking the $\sigma_{n}^{x} \rightarrow-\sigma_{n}^{x}$ symmetry of the Hamiltonian is present. When 


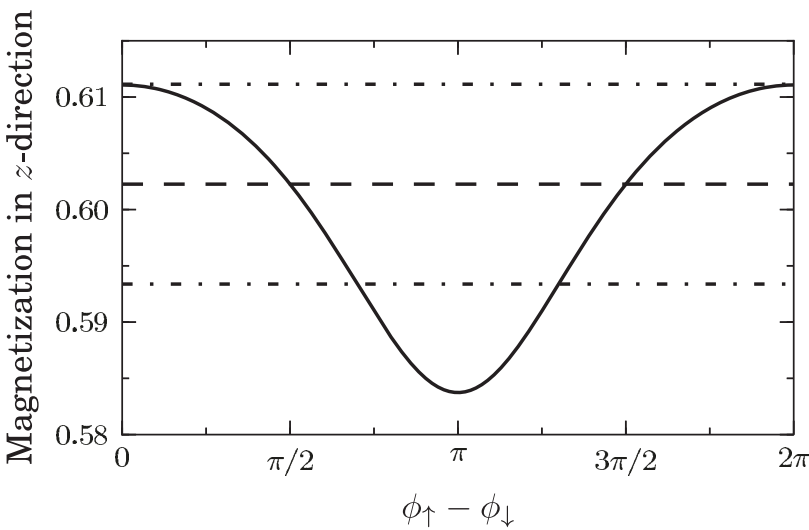

Figure 3 Mean magnetization along the z-direction in a quantum superposition of different phases, $\hat{O}=\hat{M}_{z}=\sigma_{n}^{z}$. The plot shows exact results obtained from expressions listed in the Methods section. The solid line shows $M_{z}$ evaluated from Eq. (6), the dashed line shows $\overline{M_{z}}=M_{z}^{s}$, and the dashed-dotted line shows $\overline{M_{z}} \pm \sqrt{\operatorname{var}\left(M_{z}\right)}$. We assumed $N=100, \delta=$ $0.05, c_{\uparrow}=1 / 2, c_{\downarrow}=\sqrt{3 / 4}$, and $g=1$. For these parameters $\mathcal{F} \approx 0.41$, $\overline{M_{z}} \approx 6.0 \times 10^{-1}$, and $\sqrt{\operatorname{var}\left(M_{z}\right)} \approx 8.9 \times 10^{-3}$.

necessary, we thus add a $-h \sum_{n=1}^{N} \sigma_{n}^{x}$ term to $\hat{H}_{I}(g)$ and denote a ground state of the resulting Hamiltonian as $|g, h\rangle$. Without the quantum magnetic field, $\delta=0$, the Ising chain acquires macroscopic magnetization (along the direction of the symmetry breaking field $h$ ) only in the ferromagnetic phase. This magnetization can also be calculated by studying the correlation function ${ }^{15}$

$$
\lim _{R \rightarrow \infty} \sqrt{\left|\left\langle g\left|\sigma_{1}^{x} \sigma_{R}^{x}\right| g\right\rangle\right|}=\lim _{h \rightarrow 0^{+}}\left\langle g, h\left|\sigma_{n}^{x}\right| g, h\right\rangle=\left(1-g^{2}\right)^{1 / 8} .
$$

Importantly, it encodes the critical exponent $\beta=1 / 8$ (see Ref. [16]).

To study spontaneous magnetization in the presence of the superposition of ground states, we find numerically the states $|g \pm \delta, h\rangle$ using a periodic version ${ }^{17}$ of the TEBD algorithm ${ }^{18,19}$. Then, we calculate $M_{x}^{ \pm \pm}=\left.\left\langle g \pm \delta, h\left|\sigma_{n}^{x}\right| g \pm \delta, h\right\rangle\right|_{h \approx 0}$ and $M_{x}^{+-}=\left\langle g+\delta, h\left|\sigma_{n}^{x}\right|\right.$

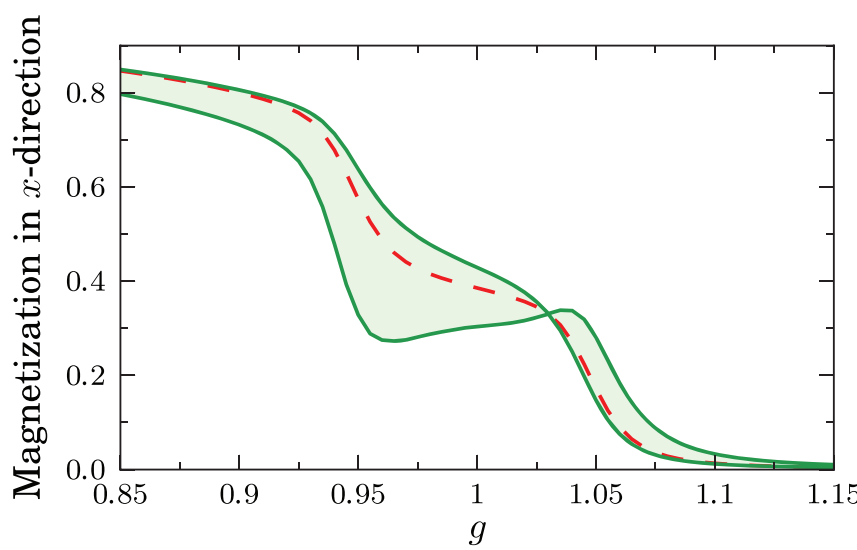

Figure $4 \mid$ Spontaneous magnetization in the $x$-direction in a quantum superposition of different phases. The red dashed line is the "standard" average $M_{x}^{s}=c_{\uparrow}^{2} M_{x}^{++}+c_{\downarrow}^{2} M_{x}^{--}$. The shaded area between the solid green lines marks the range of variation of $M_{x}$ due to variation of the relative phase $\phi_{\uparrow}-\phi_{\downarrow}$ (similar variation, but at a single magnetic field $g$, is depicted in Fig. 3). This is a numerical result obtained with the "periodic" TEBD algorithm for $\delta=0.05, h=0.0001, c_{\uparrow}=c_{\downarrow}=1 / \sqrt{2}, N=100$, and $\chi=50$ (the cut-off parameter of the algorithm). The spontaneous magnetization does not disappear for $g>1+\delta$, when both states in the superposition are in the paramagnetic phase, due to the non-zero symmetry-breaking field $h$ (see Fig. 5 for the $h \rightarrow 0^{+}$and $N \gg 1$ limits; see the Methods section for details). $g-\delta, h\rangle\left.\right|_{h \approx 0}$. Naturally, for large enough systems, the standard result is reproduced by numerics:

$$
M_{x}^{ \pm \pm} \simeq\left[1-(g \pm \delta)^{2}\right]^{1 / 8}
$$

for $|g \pm \delta|<1$ and zero otherwise. The results of TEBD calculations are plotted in Fig. 4 . The presence of the cross term magnetization, resulting from the superposition of two ground states in Eq. (5), leads to sizable deviations from the "standard" average.

To analyze this deviation more efficiently in the thermodynamic limit, we study the asymptotic behavior of the two-point correlation functions:

$$
M_{x \mathcal{F}}^{+-}=\lim _{R \rightarrow \infty} \sqrt{\left|\left\langle\sigma_{1}^{x} \sigma_{1+R}^{x}\right\rangle_{\mathcal{F}}^{+-}\right|}
$$

where $\langle\cdots\rangle_{\mathcal{F}}^{+-}=\langle g+\delta|\cdots| g-\delta\rangle / \mathcal{F}$. It can be done using the exact solution of the Ising model through fermionization, where we express the correlator as a determinant of a $2 R \times 2 R$ block Toeplitz matrix, which is then numerically evaluated (see the Methods section for details).

As shown in Fig. 5, we find that the scaling of $M_{x}^{+-}$around the critical point is consistent with the ansatz

$$
M_{x}^{+-}=\mathcal{F} \delta^{\beta} B(c), \quad c=\frac{g-g_{c}}{\delta},
$$

where $\beta=1 / 8$ and $g_{c}=1$ for the Ising chain that we study, and $B(c)$ is the scaling function. It is nonzero when at least one of the superposed ground states is in the ferromagnetic phase, i.e., $B(c) \neq 0$ for $c<1$. Far away from the critical point, we observe that $M_{x}^{++} \approx M_{x}^{--} \approx$ $M_{x \mathcal{F}}^{+-}$and so $B(c \ll-1) \simeq(-2 c)^{1 / 8}$.

\section{Discussion}

We have seen that the presence of the quantum external field allows for creation of the superposition state of two distinct ground states in general and two distinct phases in particular. If this happens,
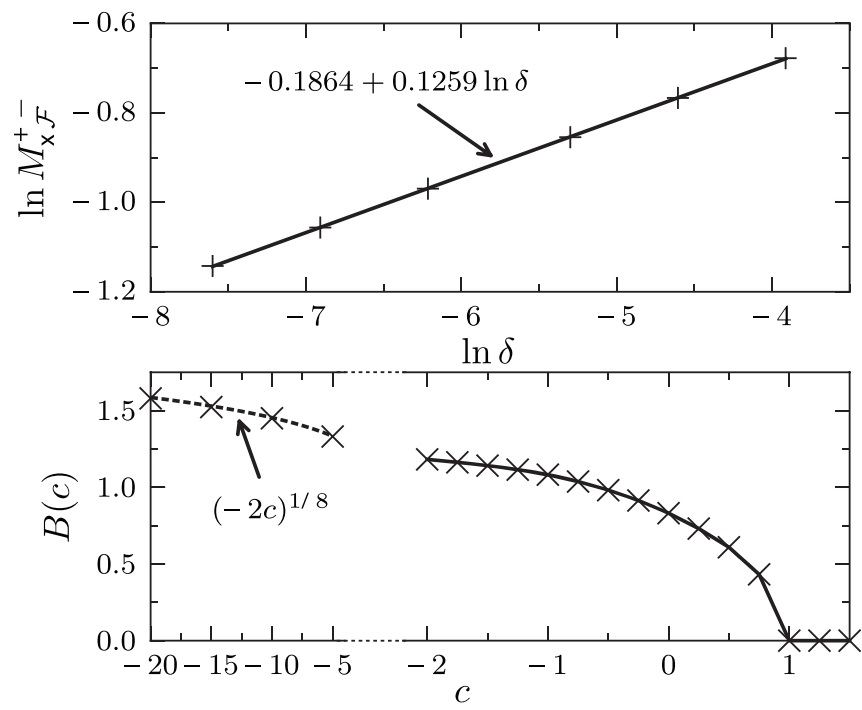

Figure 5 Scaling properties of the spontaneous magnetization cross term in the $\boldsymbol{x}$-direction (9). Upper panel: Illustration that $M_{x \mathcal{F}}^{+-}(g, \delta)$ at the critical point $g=g_{c}=1$ scales as $\delta^{1 / 8}$. Crosses show numerics based on Eq. (8), while the straight line is a fit. The same result is obtained near the critical point for other $c=\left(g-g_{c}\right) / \delta$ and $g<1+\delta$. Lower panel: Illustration of the scaling function $B(c)$ near the critical point and far away from it. Crosses show numerics, the solid line connects them, and the dashed line is $(-2 c)^{1 / 8}$. See the Methods section for details. 
expectation values are altered by the cross terms. The magnitude of this effect can be sizable as depicted in Figs. 3 and 4.

A fundamentally important question can now be answered: What is the role of the system size in a quantum phase transition in a quantum field and what critical information is imprinted onto the cross terms.

To answer it, we note that all the cross terms that we studied are a product of the two terms: The ground state fidelity $\mathcal{F}(g, \delta)$ and a term that has a well-defined non-zero value in the thermodynamic limit. Ground state fidelity, however, typically disappears in the thermodynamic limit of $N \rightarrow \infty$ often invoked in the context of quantum phase transitions.

This is known as Anderson orthogonality catastrophe after the seminal work reported in Ref. [20]. Therefore, we are interested in the studies of systems for which $N \gg 1$ (to see quantum criticality), but still $N<\infty$ (to avoid the catastrophe). There are three options here, which we will discuss below. Instead of providing specific results for fidelity of the Ising chain, we provide general scaling results to highlight the role of critical exponents in our problem and to keep the discussion concise.

First, one can consider the limit of $\delta \rightarrow 0$ taken at fixed $N \gg 1$. Then, fidelity reads $\mathrm{s}^{21-23}$

$$
\ln \mathcal{F}(g, \delta) \sim-\delta^{2} N^{2 / d v}
$$

near the critical point. Here, $d$ is system dimensionality and $v$ is the critical exponent (correlation length diverges as $\left|g-g_{c}\right|^{-v}$ near the critical point; $d=v=1$ in the Ising chain that we consider). Since fidelity is close to unity in this limit, the cross terms do not get small. One must remember, however, that they will be dominated by finite system size corrections requiring a separate study, which is beyond the scope of this work.

Second, in the limit of $N \rightarrow \infty$ at fixed $\delta$ - the one that we assumed in our calculations - one can focus on the "moderately" large systems. To explain this term we note that near the critical point (in the above-mentioned limit) ${ }^{24}$

$$
\ln \mathcal{F}(g, \delta) \sim-N \delta^{d v}
$$

The crossover from Eq. (10) to Eq. (11) happens near the critical point when ${ }^{24}$

$$
N \delta^{d v} \sim 1
$$

We define the "moderately" large system to be just large enough to exhibit the scaling of fidelity with $\delta$ and $N$ given by Eq. (11) rather than Eq. (10). In the Ising case, Eq. (11) predicts $\ln \mathcal{F} \sim-N \delta$ while Eq. (10) predicts $\ln \mathcal{F} \sim-N^{2} \delta^{2}$. For a "moderately" large system fidelity shall not be too small to erase the contribution of the cross term (see, e.g., Fig. 4).

Third, one can study superpositions of two ground states from the same phase far away from the critical point. There $\ln \mathcal{F} \sim$ $-N \delta^{2}\left|g-g_{c}\right|^{d v-2}$, and for $N \gg 1$ fidelity can be kept close to unity by a proper choice of $\delta$. The downside of this scenario is that we loose the possibility to superimpose two phases.

From the above discussion, we see that the critical exponent $v$ is imprinted onto the cross term via fidelity. Also the critical exponent $\beta$ is seen in the cross term $M_{x}^{+-}$contributing to spontaneous magnetization. The location of the critical point is most directly seen in the cross terms $M_{z}^{+-}$and $C_{x}^{+-}$through "divergence" of their second derivative over $g$ taken at $g_{c} \pm \delta$. This is caused by the singularity of the second derivative of the ground state energy per spin across the critical point. This singularity will be rounded off in finite systems $(N<\infty)$, but nevertheless there shall be pronounced peaks visible. We also note that while the "standard" averages $M_{z}^{s}$ and $C_{x}^{s}$ also encode the position of the critical point, they do not encode the critical exponent $v$.

To observe the superposed phases, experiments will have to keep decoherence to a minimum. The decoherence rate of the state in Eq.
(5) will depend on how well the environment distinguishes the two components, which will depend on the system size (see, e.g., Ref. [25]) and the overlap between the two states (fidelity). Thus, $N$ cannot be too large and $\mathcal{F}$ cannot be too small. This is a similar issue to being able to observe the effect of the cross term, which we discussed above. We thus do not expect decoherence to be overwhelming in a properly prepared setup. Further, the system size can be used as a parameter controlling decoherence, and its manipulation should be sufficient to bring decoherence down to an acceptable level. Looking from a different perspective, studies of decoherence of such a novel macroscopic quantum superposition are fundamentally interesting on its own, e.g., to boost understanding of the quantum-to-classical transition.

To conclude, we considered a quantum phase transition of an Ising chain exposed to a quantum external field. This scenario can be used to create a new state of matter where the system is simultaneously in two distinct quantum phases. Observables on the chain then take on forms that encode the ground state fidelity, the location of the critical point, and the universal critical exponents of the system. These findings set the foundations for developing a scaling theory of quantum phase transitions in quantum fields. Recent advances in cold atom cavity-QED and ion traps may lead to experimental realization of superposed phases.

\section{Methods}

We provide here some technical details regarding our calculations.

The Ising Hamiltonian $\hat{H}_{I}(g)$ is diagonalized using the standard approach (see e.g. Ref. [26]). The Jordan-Wigner transformation,

$$
\sigma_{n}^{z}=1-2 \hat{c}_{n}^{\dagger} \hat{c}_{n}, \quad \sigma_{n}^{x}=\left(\hat{c}_{n}+\hat{c}_{n}^{\dagger}\right) \prod_{m<n}\left(1-2 \hat{c}_{m}^{\dagger} \hat{c}_{m}\right),
$$

where $\hat{c}_{n}$ are fermionic annihilation operators, transforms the Ising chain to a freefermion model. After applying the Fourier transform

$$
\hat{c}_{n}=\frac{e^{-i \pi / 4}}{\sqrt{N}} \sum_{k} \hat{c}_{k} e^{i k n},
$$

the Hamiltonian takes the form:

$$
\begin{aligned}
\hat{H}_{I}(g) & =\sum_{k}\left(2 \hat{c}_{k}^{\dagger} \hat{c}_{k}-1\right)(g-\cos k)+\left(\hat{c}_{k}^{\dagger} \hat{c}_{-k}^{\dagger}+\hat{c}_{-k} \hat{c}_{k}\right) \sin k, \\
k & = \pm(2 s+1) \frac{\pi}{N}, \quad s=0, \ldots, \frac{N}{2}-1 .
\end{aligned}
$$

Diagonalization of the Hamiltonian with the help of the Bogolubov transformation leads to the following ground state wave-function

$$
|g \pm \delta\rangle=\prod_{k>0}\left[\cos \left(\theta_{k}^{ \pm} / 2\right)\left|0_{k} 0_{-k}\right\rangle-\sin \left(\theta_{k}^{ \pm} / 2\right)\left|1_{k} 1_{-k}\right\rangle\right],
$$

where $\left|m_{k}, m_{-k}\right\rangle$ describes the state with $m=0,1$ pairs of $c_{k}$ quasiparticles with momentum $k$ and

$$
\tan \theta_{k}^{ \pm}=\frac{\sin k}{g \pm \delta-\cos k} .
$$

To prepare Fig. 3, we fix the system size $N$ and use the following exact expressions for magnetization and fidelity

$$
\begin{aligned}
& M_{z}^{ \pm \pm}=\left\langle g \pm \delta\left|\sigma_{n}^{z}\right| g \pm \delta\right\rangle=\frac{1}{N} \sum_{k} \cos \theta_{k}^{ \pm} \\
& M_{z}^{+-}=\left\langle g+\delta\left|\sigma_{n}^{z}\right| g-\delta\right\rangle=\frac{\mathcal{F}}{N} \sum_{k} \frac{\cos \frac{\theta_{k}^{+}+\theta_{k}^{-}}{2}}{\cos \frac{\theta_{k}^{+}-\theta_{k}^{-}}{2}} \\
& \mathcal{F}=\langle g+\delta \mid g-\delta\rangle=\prod_{k>0} \cos \left(\frac{\theta_{k}^{+}-\theta_{k}^{-}}{2}\right)>0
\end{aligned}
$$

To prepare Fig. 4 , we select $g, \delta$ and $h$, and calculate the ground states $|g \pm \delta, h\rangle$ of the Ising chain exposed to transverse and longitudinal magnetic fields. This is done through imaginary time evolution performed with the periodic TEBD algorithm. A global phase of the wave-functions is then chosen to make $\mathcal{F}=\langle g-\delta, h \mid g+\delta, h\rangle$ positive. We then directly calculate $M_{x}^{ \pm \pm}$and $M_{x}^{+-}$(both are positive). Putting these results into Eq. (6), one can calculate the spontaneous magnetization in the $x$ direction in the superposition state (5). The result is still dependent on the relative phase $\phi_{\uparrow}-\phi_{\downarrow}$. When this phase is either 0 or $\pm \pi$, spontaneous magnetization at any fixed $g, \delta$, and $h$ reaches an extremum. These extremal values are depicted by solid green lines in Fig. 4. 
To prepare Fig. 5, we calculate correlation function

$$
C_{x x}^{+-}(R)_{\mathcal{F}}=\left|\left\langle\sigma_{1}^{x} \sigma_{1+R}^{x}\right\rangle_{\mathcal{F}}^{+-}\right|=\left|\left\langle\Pi_{i=1}^{R} \hat{b}_{i} \hat{a}_{i+1}\right\rangle_{\mathcal{F}}^{+-}\right|,
$$

where we introduce $\hat{b}_{n}=\hat{c}_{n}^{\dagger}-\hat{c}_{n}$ and $\hat{a}_{n}=\hat{c}_{n}^{\dagger}+\hat{c}_{n}$. We study it, because $M_{x \mathcal{F}}^{+-}=$ $\lim _{R \rightarrow \infty} \sqrt{C_{x x}^{+-}(R)_{\mathcal{F}}}$.

The next step is to use Wick's theorem extended to such a cross-correlation ${ }^{27}$. It can be used as long as the overlap $\mathcal{F}$ is nonzero, which is the case in our calculations. Then extending the results of Ref. [28], we find that $C_{x x}^{+-}(R)_{\mathcal{F}}$ can be expressed as a Pfaffian of a $2 R \times 2 R$ antisymmetric matrix, which can be converted into a determinant:

$$
\begin{aligned}
& C_{x x}^{+-}(R)_{\mathcal{F}}=\sqrt{\operatorname{det}\left[A_{R}\right]}, \\
& A_{R}=\left[\begin{array}{cc}
\left\langle\hat{b}_{m} \hat{b}_{n}\right\rangle_{\mathcal{F}}^{+-} & \left\langle\hat{b}_{m} \hat{a}_{n+1}\right\rangle_{\mathcal{F}}^{+-} \\
\left\langle\hat{a}_{m+1} \hat{b}_{n}\right\rangle_{\mathcal{F}}^{+-} & \left\langle\hat{a}_{m+1} \hat{a}_{n+1}\right\rangle_{\mathcal{F}}^{+-}
\end{array}\right]_{m, n=1 \ldots R},
\end{aligned}
$$

where $A_{R}$ is a block Toeplitz matrix. Apart from a few special cases, it is not known how to calculate such a determinant analytically $y^{29}$. Thus, we use numerics with a large enough $R$ to obtain a well-converged result. We employ a continuous (i.e. $N \rightarrow \infty$ ) approximation for the elements of the Toeplitz matrix

$$
\begin{aligned}
& \left\langle\hat{a}_{m} \hat{a}_{n}\right\rangle_{\mathcal{F}}^{+-}=\frac{-i}{2 \pi} \int_{-\pi}^{\pi} d k \tan \frac{\theta_{k}^{+}-\theta_{k}^{-}}{2} e^{i k(m-n)}, \\
& \left\langle\hat{b}_{m} \hat{a}_{n}\right\rangle_{\mathcal{F}}^{+-}=\frac{-1}{2 \pi} \int_{-\pi}^{\pi} d k \frac{e^{-i\left(\theta_{k}^{+}+\theta_{k}^{-}\right) / 2}}{\cos \frac{\theta_{k}^{+}-\theta_{k}^{-}}{2}} e^{i k(m-n)},
\end{aligned}
$$

and $\left\langle\hat{a}_{m} \hat{a}_{n}\right\rangle_{\mathcal{F}}^{+-}=\left\langle\hat{b}_{m} \hat{b}_{n}\right\rangle_{\mathcal{F}}^{+-},\left\langle\hat{a}_{m} \hat{b}_{n}\right\rangle_{\mathcal{F}}^{+-}=-\left\langle\hat{b}_{n} \hat{a}_{m}\right\rangle_{\mathcal{F}}^{+-}$. Regarding the para-

meter $R$, we mention that it has to be of the order of 500 (2000) for $g=0.995$ and $\delta=$ $0.01(g=1.005$ and $\delta=0.01)$ in order for the results to be converged to the $R \rightarrow \infty$ limit. For every $g$ and $\delta$ sufficiently large $R$ is chosen to calculate data for Fig. 5 .

We also mention that we verified the Pfaffian-based numerics with a direct numerical calculation using the TEBD algorithm. For systems composed of about 100 spins, for which the TEBD algorithm can still be efficiently applied, spontaneous magnetization from both calculations agree.

Finally, we provide definition of the elliptic functions that we use in the Results section:

$$
K(x)=\int_{0}^{\pi / 2} \frac{d \phi}{\sqrt{1-x \sin ^{2} \phi}}, E(x)=\int_{0}^{\pi / 2} d \phi \sqrt{1-x \sin ^{2} \phi} .
$$

1. Sachdev, S. Quantum Phase Transitions (Cambridge University Press, Cambridge, U.K., 2011).

2. Lewenstein, M., Sanpera, A., Ahufinger, V., Damski, B., Sen(De), A. \& Sen, U. Ultracold atomic gases in optical lattices: mimicking condensed matter physics and beyond. Adv. Phys. 56, 243 (2007).

3. Maschler, C. \& Ritsch, H. Cold atom dynamics in a quantum optical lattice potential. Phys. Rev. Lett. 95, 260401 (2005).

4. Larson, J., Fernández-Vidal, S., Morigi, G. \& Lewenstein, M. Quantum stability of Mott-insulator states of ultracold atoms in optical resonators. New J. Phys. 10, 045002 (2008)

5. Brennecke, F., Donner, T., Ritter, S., Bourdel, T., Köhl, M. \& Esslinger, T. Cavity QED with a Bose-Einstein condensate. Nature 450, 268 (2007).

6. Hanson, R., Dobrovitski, V. V., Feiguin, A. E., Gywat, O. \& Awschalom, D. D. Coherent dynamics of a single spin interacting with an adjustable spin bath. Science 320, 352 (2008).

7. Bluhm, H. et al. Dephasing time of GaAs electron-spin qubits coupled to a nuclear bath exceeding $200 \mu \mathrm{s}$. Nature Physics 7, 109 (2011).

8. Cywiński, Ł. Dephasing of electron spin qubits due to their interaction with nuclei in quantum dots. Acta Phys. Pol. A 119, 576 (2011).
9. Zhang, J., Peng, X., Rajendran, N. \& Suter, D. Detection of quantum critical points by a probe qubit. Phys. Rev. Lett. 100, 100501 (2008).

10. Porras, D. \& Cirac, J. I. Effective quantum spin systems with trapped ions. Phys. Rev. Lett. 92, 207901 (2004).

11. Korenblit, S. et al. Quantum simulation of spin models on an arbitrary lattice with trapped ions. e-print arXiv:1201.0776 (2012).

12. Islam, R. et al. Onset of a quantum phase transition with a trapped ion quantum simulator. Nature Communications 2, 377 (2011).

13. Damski, B., Quan, H. T. \& Zurek, W. H. Critical dynamics of decoherence. Phys. Rev. A 83, 062104 (2011)

14. Gu, S.-J. Fidelity approach to quantum phase transitions. Int. J. Mod. Phys. B $\mathbf{2 4}$, 4371 (2010).

15. Pfeuty, P. The one-dimensional Ising model with a transverse field. Ann. Phys. 57, 79 (1970).

16. Continentino, M. A. Quantum Scaling in Many-Body Systems (World Scientific Publishing, Singapore, 2001).

17. Danshita, I. \& Naidon, P. Bose-Hubbard ground state: Extended Bogoliubov and variational methods compared with time-evolving block decimation. Phys. Rev. A 79, 043601 (2009).

18. Vidal, G. Efficient classical simulation of slightly entangled quantum computations. Phys. Rev. Lett. 91, 147902 (2003).

19. Vidal, G. Efficient simulation of one-dimensional quantum many-body systems. Phys. Rev. Lett. 93, 040502 (2004).

20. Anderson, P. W. Infrared catastrophe in Fermi gases with local scattering potentials. Phys. Rev. Lett. 18, 1049 (1967).

21. Albuquerque, A. F., Alet, F., Sire, C. \& Capponi, S. Quantum critical scaling of fidelity susceptibility. Phys. Rev. B 81, 064418 (2010).

22. Barankov, R. A. Quench dynamics as a probe of quantum criticality. e-print arxiv:0910.0255 (2009).

23. Gritsev, V. \& Polkovnikov, A. Universal dynamics near quantum critical points. e-print arXiv:0910.3692 (2009).

24. Rams, M. M. \& Damski, B. Quantum fidelity in the thermodynamic limit. Phys. Rev. Lett. 106, 055701 (2011).

25. Dziarmaga, J., Zurek, W. H. \& Zwolak, M. Non-local quantum superpositions of topological defects. Nature Physics 8, 49 (2012).

26. Dziarmaga, J. Dynamics of a quantum phase transition: Exact solution of the quantum Ising model. Phys. Rev. Lett. 95, 245701 (2005).

27. Balian, R. \& Brezin, E. Nonunitary Bogoliubov transformations and extension of Wick's theorem. Nuovo Cimento 64, 37 (1969).

28. Barouch, E. \& McCoy, B. M. Statistical mechanics of the XY model. II. Spincorrelation functions. Phys. Rev. A 3, 786 (1971).

29. Its, A. R. \& Korepin, V. E. The Fisher-Hartwig formula and entanglement entropy. J. Stat. Phys. 137, 1014 (2009)

\section{Acknowledgements}

This work is supported by U.S. Department of Energy through the LANL/LDRD Program. MMR acknowledges support from the FWF SFB grant F4014.

\section{Author contributions}

All authors contributed to the research described in this manuscript and to its preparation.

\section{Additional information}

Competing financial interests: The authors declare no competing financial interests.

License: This work is licensed under a Creative Commons

Attribution-NonCommercial-NoDerivative Works 3.0 Unported License. To view a copy of this license, visit http://creativecommons.org/licenses/by-nc-nd/3.0/

How to cite this article: Rams, M.M., Zwolak, M. \& Damski, B. A quantum phase transition in a quantum external field: Superposing two magnetic phases. Sci. Rep. 2, 655;

DOI:10.1038/srep00655 (2012). 